\title{
A Fault Feature Extraction Method for Rolling Bearing Based on Pulse Adaptive Time-Frequency Transform
}

\author{
Jinbao Yao, ${ }^{1}$ Baoping Tang, ${ }^{1}$ and Jie Zhao ${ }^{2}$ \\ ${ }^{1}$ State Key Lab of Mechanical Transmission, Chongqing University, Chongqing 400030, China \\ ${ }^{2}$ College of Mechanical Engineering, Chengdu University, Sichuan 610106, China \\ Correspondence should be addressed to Jinbao Yao; jbyao2001@163.com
}

Received 18 December 2015; Revised 27 February 2016; Accepted 2 March 2016

Academic Editor: Juan P. Amezquita-Sanchez

Copyright (c) 2016 Jinbao Yao et al. This is an open access article distributed under the Creative Commons Attribution License, which permits unrestricted use, distribution, and reproduction in any medium, provided the original work is properly cited.

\begin{abstract}
Shock pulse method is a widely used technique for condition monitoring of rolling bearing. However, it may cause erroneous diagnosis in the presence of strong background noise or other shock sources. Aiming at overcoming the shortcoming, a pulse adaptive time-frequency transform method is proposed to extract the fault features of the damaged rolling bearing. The method arranges the rolling bearing shock pulses extracted by shock pulse method in the order of time and takes the reciprocal of the time interval between the pulse at any moment and the other pulse as all instantaneous frequency components in the moment. And then it visually displays the changing rule of each instantaneous frequency after plane transformation of the instantaneous frequency components, realizes the time-frequency transform of shock pulse sequence through time-frequency domain amplitude relevancy processing, and highlights the fault feature frequencies by effective instantaneous frequency extraction, so as to extract the fault features of the damaged rolling bearing. The results of simulation and application show that the proposed method can suppress the noises well, highlight the fault feature frequencies, and avoid erroneous diagnosis, so it is an effective fault feature extraction method for the rolling bearing with high time-frequency resolution.
\end{abstract}

\section{Introduction}

As the most common part in the rotating machinery, rolling bearing is most vulnerable to damage. The condition of the rolling bearing has a great effect on the working condition of the whole machine. Any defect in the rolling bearing may impair the normal working order of the machine, causing vibration in the machine, producing noises, and even resulting in fatal crashes. Therefore, it is necessary to perform fault diagnosis for defects in the rolling bearing. A damaged rolling bearing may produce regular shock pulses, and these components may be contained in the vibration signals. Besides, the shock modulation phenomenon may take place. However, as the operating condition of the rolling bearing is quite complex, in addition to the shock pulse components representing the fault information, the vibration signals produced by the rolling bearing always contain other signal components such as noise, rotation frequency, and its harmonic components, which usually interfere with the fault diagnosis of the rolling bearing. Hence, the key of the rolling bearing fault diagnosis is to extract from the vibration signals the shock pulse representing the damage. Considering that the shock modulation is an important feature when any fault arises in the rolling bearing, the fault feature frequency can be extracted by demodulating the vibration signals, so as to realize the fault diagnosis of the rolling bearing. So the study on the vibration signal demodulation methods for the rolling bearing has for long become an attraction and also a difficulty in the area of rolling bearing fault diagnosis.

Present vibration signal demodulation methods for the rolling bearing mainly include the envelope demodulation method, resonance demodulation method (RDM), and shock pulse method (SPM). The envelope demodulation method uses a high frequency vibration sensor, acquires the high frequency vibration signals of the rolling bearing containing the low frequency shock components by setting a certain high frequency filtering bandwidth, and performs such processes as envelope interception and capture, demodulation, and low-pass filtering, so as to obtain the modulation frequency (rolling bearing fault feature frequency). 
It mainly includes the generalized detection-filtering demodulation method [1], local mean decomposition demodulation method [2], wavelet analysis demodulation method [3-5], EMD/EEMD demodulation method [6], and Hilbert demodulation method $[7,8]$. Although the envelope demodulation methods in question have produced some results in the application study on the rolling bearing fault diagnosis, owing to their common limitations, the demodulated low frequency shock signals representing the fault information always contain noise and information of other vibration sources such as rotation axis and gear, which may badly interfere with the rolling bearing fault diagnosis, and the frequency domain information of the demodulation wave contains the frequency components that cannot be analyzed or may cause erroneous diagnosis. In addition, if the signal-to-noise ratio (SNR) of the shock signal is not raised, the envelope demodulation methods cannot be applied to the early fault diagnosis of the rolling bearing. Following the phenomenon that the shock signal of wide range may arouse resonance, and the vibration signal rarely produces resonance [9], SPM and RDM both modulate the low frequency shock signal to high frequency one, perform such processes as filtering and envelope demodulation, and then diagnose the working condition of the rolling bearing. Although both methods above solve the problems in the envelope demodulation method, they still have their own problems due to differences in the working principle. RDM modulates the shock signal to the resonance frequency of the mechanical system structure and employs the frequency domain information of the resonance demodulation wave to diagnose the working condition of the rolling bearing $[10,11]$. It is able to accurately diagnose the faulty parts. But as the resonance frequency varies with the different mechanical system structures, it is hard for the method to select the filtering center frequency and bandwidth. And SPM modulates the shock signal to a fixed high frequency and uses the amplitude information of the resonance demodulation wave to diagnose the working condition of the rolling bearing $[12,13]$. Though the method does not have the problem of RDM whose filtering center frequency and bandwidth can be hardly selected, as it adopts the fixed filtering center frequency and bandwidth, performs the envelope detection which is characterized by nonlinear transformation, and bases the diagnosis on the amplitude information of the resonance demodulation wave alone, without any further analysis or processing, it cannot arrive at desired diagnosis results in the presence of the strong background noise or other shock sources. To solve the problems as mentioned, the researchers proposed some improved methods such as shock pulse frequency analysis method [14], shock pulse time-frequency analysis method, and improved redundant lifting scheme method [15], but they just used the existing signal processing methods for analyzing frequency spectrum, time-frequency spectrum, and the like. Such proposed methods are still not suitable for the rolling bearing fault diagnosis in the presence of strong background noise, and they also lose the instantaneous time change feature of the shock.

For this reason, by integrating the advantages of RDM and SPM, this paper comes up with a pulse adaptive

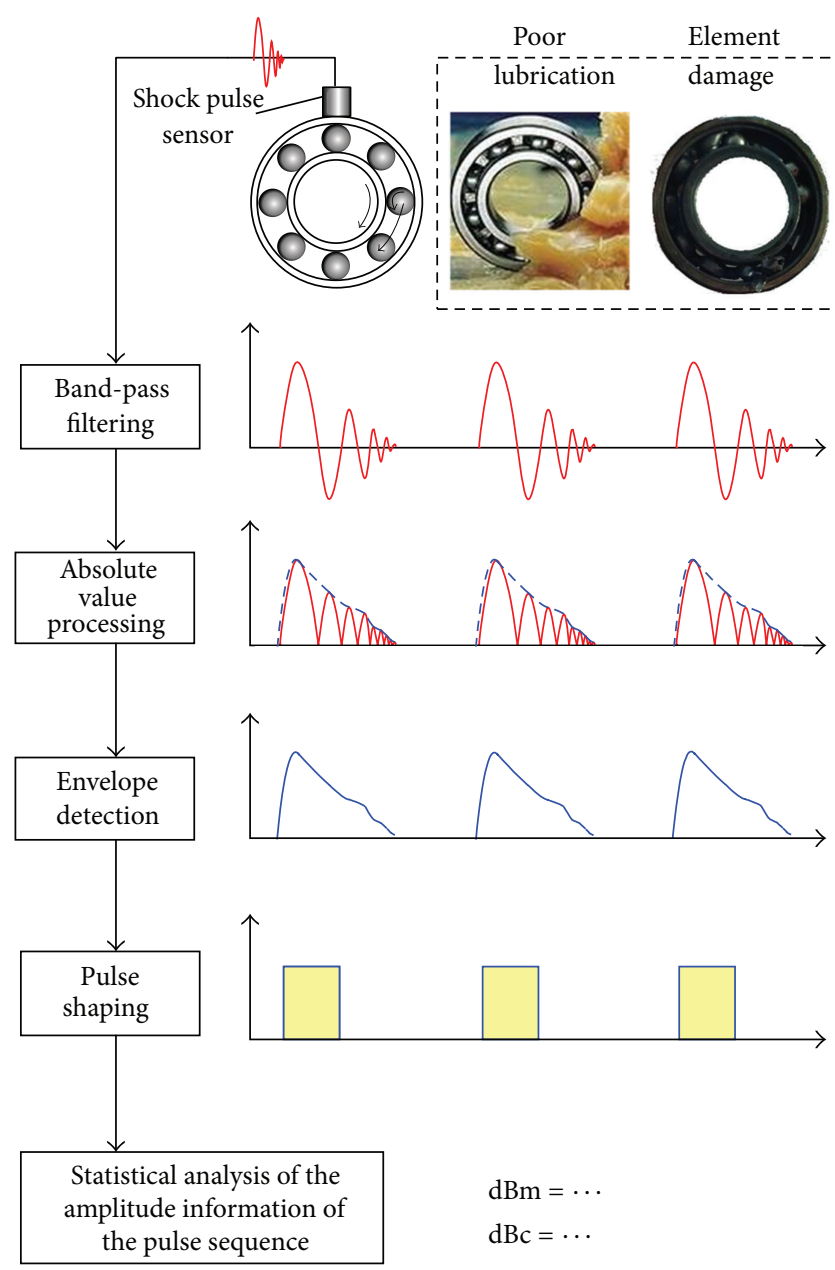

FIGURE 1: Block diagram of working principle of SPM.

time-frequency transform method (PATFTM). Like SPM, the proposed method uses the fixed filtering center frequency and bandwidth; and also like RDM, it is able to further process the extracted shock pulse, thus avoiding the problem existing in SPM.

The remainder of the paper is organized as follows: the working principle and diagnosis method of SPM are introduced in Section 2; on the basis of SPM, Section 3 proposes the PATFTM; through simulation and application analysis, PATFTM is validated in Section 4; and finally, based on the analysis results, Section 5 draws the study conclusions and makes suggestions for further studies in this regard.

\section{Shock Pulse Method}

SPM was first proposed in the 1970s [16], for diagnosing the failures in the rolling bearing, with its working principle shown in Figure 1. The rolling bearing in service, due to such reasons as poor lubrication, surface roughness, and element damage, may cause shock effect. And together with the mechanical and electrical tuning, the dedicated shock pulse sensor may be driven to resonate at a fixed frequency (usually within the range of $20 \mathrm{kHz} \sim 60 \mathrm{kHz}$ ) [17], so that the resonance signal amplitude acquired is obviously greater 


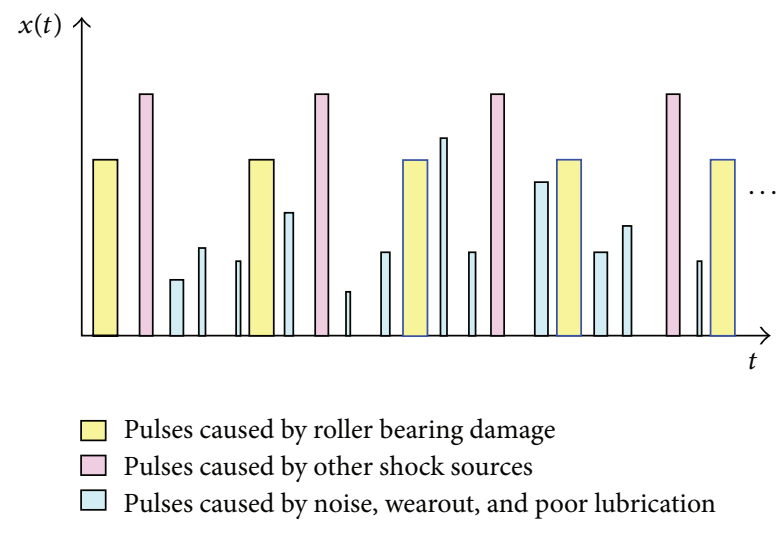

FIGURE 2: Schematic diagram of shock pulses extracted by SPM.

than the vibration signal amplitude collected by a common sensor. Then, the acquired resonance signals containing the amplitude modulation wave of the low frequency shock, random interference, and other shock sources are made to undergo such processes as band-pass filtering, absolute value processing, envelope detection, and pulse shaping, to obtain the pulse sequence. And the amplitude information of the pulse sequence is statistically analyzed, to finally diagnose the working condition of the rolling bearing.

After the pulse sequence is obtained, the statistical analysis of the amplitude information of the pulse sequence is the core of SPM. The statistical analysis means that dB-Maximum Value $(\mathrm{dBm})$ and $\mathrm{dB}$-Carpet Value $(\mathrm{dBc})$, two physical quantities used for measuring the shock pulse amplitude, are defined, recorded, and analyzed, and then, according to the diagnosis rule of SPM, the failures in the rolling bearing are diagnosed. $\mathrm{dBm}$ denotes the strong pulse decibel value and corresponds to the low frequency pulse sequence amplitude. It is defined as the maximum shock peak value obtained with a single testing period and used for measuring the extent of damage of the rolling bearing component. $\mathrm{dBc}$ denotes the carpet decibel value and corresponds to the high frequency pulse sequence amplitude. It is defined as the mean value of lots of weak shock signals and used for measuring the lubrication, surface roughness, and degree of wear. The diagnosis rule of SPM is described as follows: if $\mathrm{dBm}$ and $\mathrm{dBC}$ both are often very low, this indicates that the bearing is well lubricated and free of damage; if $\mathrm{dBm}$ and $\mathrm{dBc}$ both are often very high, this indicates that the bearing is poorly lubricated; if the periodic peak value occurs and $\mathrm{dBm}$ rises, this indicates that the bearing is damaged [18].

The shock pulses extracted by SPM mainly consist of the pulses caused by such factors as rolling bearing damage, wearout failure, poor lubrication, other shock sources, and noise, as shown in Figure 2. SPM is well known for its simplicity and practicality and carries out the fault diagnosis of the rolling bearing through the statistical analysis of the pulse sequence amplitude. It is very accurate in diagnosing the wearout failure, poor lubrication, and so forth, and it is able to diagnose the damage in the rolling bearing. However, it is not capable of performing further diagnosis of the bearing damage, and in the presence of strong background noise or other shock sources, it cannot arrive at desired diagnosis results [19].

Thus, for the sake of solving the problems in SPM, weeding out the pulse interference resulting from such factors as wearout failure, poor lubrication, other shock sources, and noise, extracting the pulses produced by the damage in the rolling bearing, and carrying out the further diagnosis of the damage in the rolling bearing, on the basis of SPM, PATFTM is proposed.

\section{Pulse Adaptive Time-Frequency Transform Method}

The basic idea of PATFTM is transforming the shock pulse sequence of one-dimensional time domain extracted by SPM into two-dimensional time-frequency domain, weeding out the interference from other factors through the effective instantaneous frequency extraction, highlighting the fault feature representing the rolling bearing damage, and finally realizing the fault diagnosis of the rolling bearing visually and effectively.

PATFTM mainly comprises such steps as amplitude threshold processing, shock pulse ordering, instantaneous frequency calculation, instantaneous frequency plane transformation, amplitude relevancy processing, and effective instantaneous frequency extraction, as shown in Figure 3.

In the following, with the application on the shock pulse sequence as shown in Figure 2, these processes will be discussed in detail.

(a) Amplitude Threshold Processing. In order to reduce the influences from such factors as background noise, wearout failure, and poor lubrication and raise the efficiency of PATFTM, the shock pulses extracted by SPM are made to experience the amplitude threshold processing by setting the threshold, in which the shock pulses whose amplitude is smaller than the threshold are excluded and those whose amplitude is greater than the threshold are retained. The threshold setting principle is regarding the average amplitude measured when the bearing is well lubricated and free of any damage as the threshold for amplitude threshold processing. Figure 4 shows the processing results of the shock pulse sequence as shown in Figure 2 through the amplitude threshold processing.

(b) Shock Pulse Ordering. As a basic physical quantity of communication signals, pulse arrival time is widely used in deinterleaving of radar pulses [20, 21]. Considering there is some similarity in regularity of pulse sequence between the radar pulses and the shock pulses to some extent, pulse arrival time is introduced into analyzing shock pulses. The shock pulses for which the amplitude threshold processing has been performed, as shown in Figure 4, are ranked in the order of pulse arrival time $t_{i}$, and the shock pulse sequence $\left\{x\left(t_{i}\right), i \in[1, N]\right\}$ is obtained, as shown in Figure 5. Here, $i$ denotes the serial number of the shock pulse in the sequence, $i \in[1, N] ; N$ denotes the total number of the shock pulses, namely, the total length of the sequence; $t_{i}$ denotes the arrival 


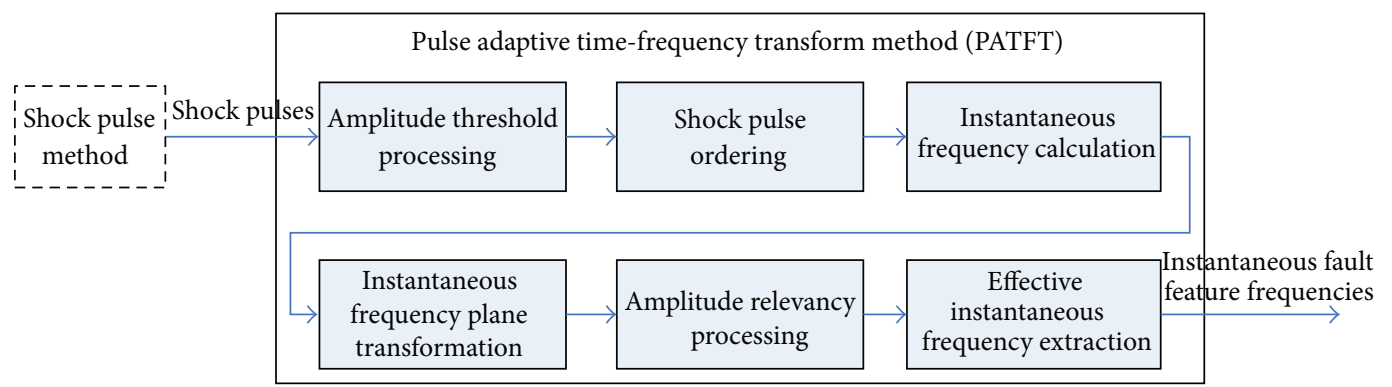

Figure 3: Steps of PATFTM.

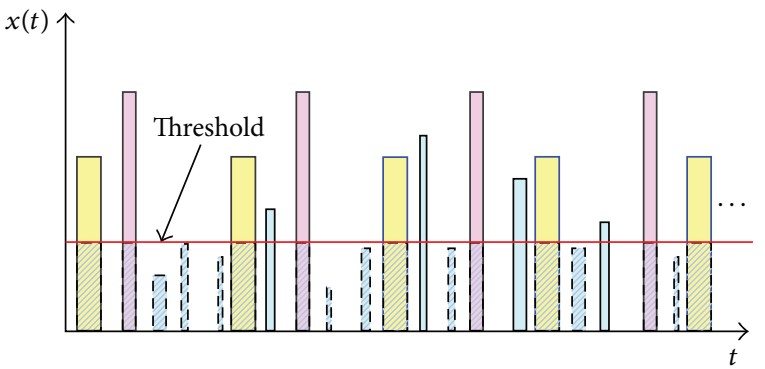

Pulses caused by roller bearing damage

Pulses caused by other shock sources

Pulses caused by noise, wearout, and poor lubrication

FIGURE 4: Schematic diagram of amplitude threshold processing.

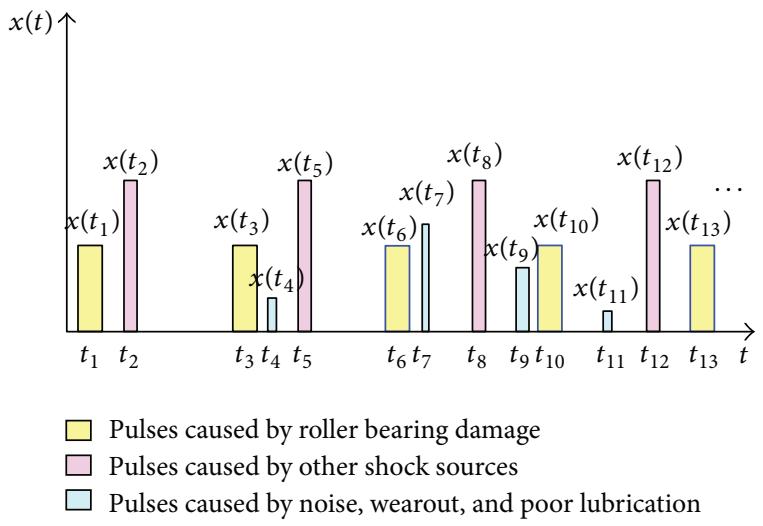

FIGURE 5: Schematic diagram of shock pulse ordering.

time of the $i$ th shock pulse, with s as its unit; $x\left(t_{i}\right)$ denotes the amplitude of the $i$ th shock pulse, with $\mathrm{m} / \mathrm{s}^{2}$ as its unit.

(c) Instantaneous Frequency Calculation. The shock pulse sequence $\left\{x\left(t_{i}\right), i \in[1, N]\right\}$ contains the shock information caused by all excitation sources, including the rolling bearing damage. The sequence has relatively high internal relevancy, and the rolling bearing fault feature cannot be accurately extracted by studying some pulse alone. Thus, this paper studies the pulse arrival time $t_{i}$ and performs overall analysis of a group of pulses, to find the internal changing rule of pulses, obtain the instantaneous frequency components contained in the moment $t_{i}$, and identify different excitation sources.

Considering that the pulse arrival times $t_{i}$ and $t_{j}$ correspond to the pulse occurrence moment, for the periodical shock sequence, if the shock at the moment $t_{i}$ is produced by the damage in a rolling bearing, and the shock at the moment $t_{j}$ is generated by the same damage in the rolling bearing next time or last time, then $\left|1 /\left(t_{j}-t_{i}\right)\right|$ may be used for representing the feature frequency of the rolling bearing damage.

Thus, by reference to the feature frequency calculated from the periodical shock sequence, the instantaneous frequency component $f_{i, j}$ that may be contained in the moment $t_{i}$ in the shock pulse sequence $\left\{x\left(t_{i}\right), i \in[1, N]\right\}$ can be calculated with the positive instantaneous frequency components expressed by (1) or the reverse instantaneous frequency components expressed by (2):

$$
\begin{aligned}
& f_{i, j} \\
& = \begin{cases}\frac{1}{t_{j}-t_{i}}, & t_{j}-t_{i} \in\left[\frac{1}{f_{u}}, \frac{1}{f_{d}}\right], i \in[1, N], j \in(i, N] \\
0, & \text { Others }\end{cases} \\
& f_{i, j} \\
& = \begin{cases}\frac{1}{t_{i}-t_{j}}, & t_{i}-t_{j} \in\left[\frac{1}{f_{u}}, \frac{1}{f_{d}}\right], i \in[1, N], j \in(1, i] \\
0, & \text { Others, }\end{cases}
\end{aligned}
$$

where $i$ and $j$ denote the serial number of the $i$ th shock pulse and the $j$ th shock pulse, respectively; $t_{i}$ and $t_{j}$ denote the arrival time of the $i$ th shock pulse and the $j$ th shock pulse, respectively, with $s$ as the unit; $f_{d}$ and $f_{u}$ denote the lower limit and the upper limit of $f_{i, j}$ (as the rolling bearing fault feature frequency can be obtained through theoretical calculation, and it tends to fall within a frequency range; the lower limit $f_{d}$ and the upper limit $f_{u}$ of $f_{i, j}$ can be defined in advance, to obtain the conditions for calculation termination of $\left.f_{i, j}\right)$, with $\mathrm{Hz}$ as the unit.

Taking the calculation of the positive and reverse instantaneous frequency components of the pulse sequence as shown in Figure 5 at the moment $t_{6}$ as an example, formulas (1) and (2) are used for the calculation, and the results obtained are shown in Figure 6.

Figure 6 clearly shows that the positive and reverse instantaneous frequency components calculated with (1) and 


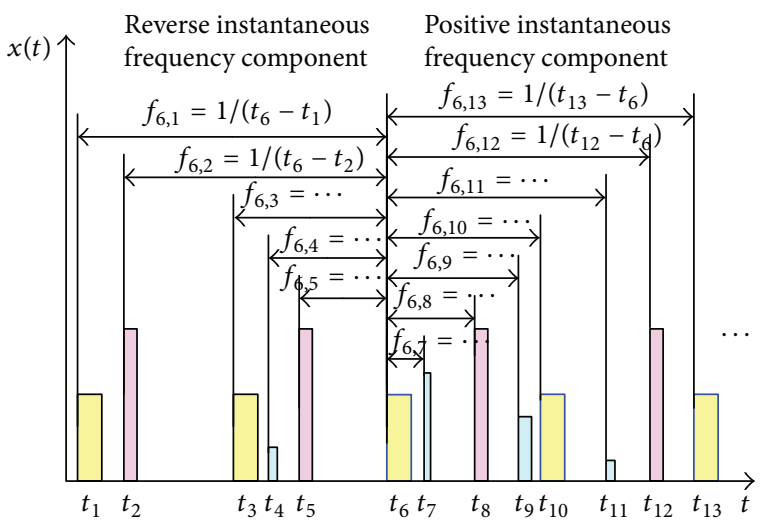

FIgURE 6: Schematic diagram of instantaneous frequency component calculation.

(2) have the instantaneous time change feature and that all instantaneous frequency components that may be contained in the shock pulse occurrence moment can be accurately and timely captured.

(d) Plane Transformation of Instantaneous Frequency. With $i$ as the row and $j$ as the column, the positive and reverse instantaneous frequency components $f_{i, j}$ both can be expressed by the matrix $F$ in

F

$$
=\left[\begin{array}{ccccccccc}
0 & f_{1,2} & \cdots & f_{1, i} & \cdots & f_{1, j} & \cdots & f_{1, N-1} & f_{1, N} \\
f_{2,1} & 0 & \cdots & f_{2, i} & \cdots & f_{2, j} & \cdots & f_{2, N-1} & f_{2, N} \\
\vdots & \vdots & \ddots & \vdots & & \vdots & & \vdots & \vdots \\
f_{i, 1} & f_{i, 2} & \cdots & 0 & \cdots & f_{i, j} & \cdots & f_{i, N-1} & f_{i, N} \\
\vdots & \vdots & \vdots & \vdots & \ddots & \vdots & & \vdots & \vdots \\
f_{j, 1} & f_{j, 2} & \cdots & f_{j, i} & \cdots & 0 & \cdots & f_{j, N-1} & f_{j, N} \\
\vdots & \vdots & & \vdots & & \vdots & \ddots & \vdots & \vdots \\
f_{N-1,1} & f_{N-1,2} & \cdots & f_{N-1, i} & \cdots & f_{N-1, j} & \cdots & 0 & f_{N-1, N} \\
f_{N, 1} & f_{N, 2} & \cdots & f_{N, i} & \cdots & f_{N, j} & \cdots & f_{N, N-1} & 0
\end{array}\right] .
$$

The elements $f_{i, j}(i=j)$ on the diagonal of the matrix $F$ all equal zero.

The elements $f_{i, j}(i \in[1, N], j \in(i, N])$ above the diagonal of the matrix $F$ correspond to the positive instantaneous frequency components and are bound by the condition $t_{i}-t_{j} \in\left[1 / f_{u}, 1 / f_{d}\right]$. With $j$ increasing, most elements above the diagonal are zero, and the number of the nonzero elements is smaller than or equal to $N-i$, and $f_{i, p}>f_{i, q}(i<p<q \leq N)$ is satisfied.

The elements $f_{i, j}(i \in[1, N], j \in(1, i])$ below the diagonal of the matrix $F$ correspond to the reverse instantaneous frequency components and are bound by the condition $t_{j}-t_{i} \in\left[1 / f_{u}, 1 / f_{d}\right]$. With $j$ decreasing, most elements below the diagonal are zero, and the number of the nonzero elements is smaller than or equal to $j-1$, and $f_{i, p}<f_{i, q}(1 \leq p<q<i)$ is satisfied.

The elements of the $i$ th row above or below the diagonal of the matrix $F$ mean the instantaneous frequency components

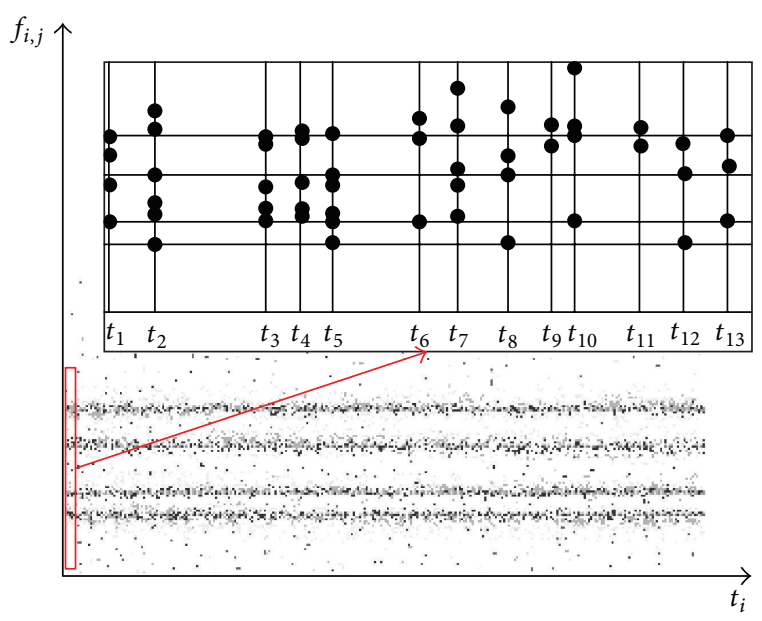

FIGURE 7: Schematic diagram of plane transformation of instantaneous frequency.

that may be contained in the vibration signal to which the shock pulse sequence $\left\{x\left(t_{i}\right), i \in[1, N]\right\}$ corresponds, at the moment $t_{i}$.

If the elements above the diagonal of the matrix $F$ (positive instantaneous frequency components) are processed by the plane transformation method applied to the communication domain $[20,21]$, they will be displayed in the plane coordinates with $t_{i}$ as horizontal coordinate and $f_{i, j}$ as vertical coordinate, and the instantaneous frequency component $f_{i, j}$ of the shock pulse sequence $\left\{x\left(t_{i}\right), i \in[1, N]\right\}$ and its changing rule will be shown with the arrival time $t_{i}$ in a dynamic manner, the process which is called the plane transformation of instantaneous frequency. If the elements below the diagonal of the matrix $F$ (reverse instantaneous frequency components) are made to experience the transformation above, the process is called the reverse plane transformation of instantaneous frequency. Figure 7 shows the results of plane transformation of instantaneous frequency of all pulse sequences as shown in Figure 5.

(e) Amplitude Relevancy Processing. After the pulse sequences extracted by SPM undergo the amplitude threshold processing, shock pulse ordering, instantaneous frequency calculation, and plane transformation of instantaneous frequency, the fault feature frequency has been extracted for qualitative fault diagnosis of the rolling bearing. However, as there is no amplitude information of all fault feature frequency components, further quantitative diagnosis and trend prediction cannot be carried out for the rolling bearing fault. Thus, the instantaneous frequency component $f_{i, j}$ needs to experience the amplitude relevancy processing.

The amplitude to which the instantaneous frequency component $f_{i, j}$ corresponds is expressed as $X\left(t_{i}, f_{i, j}\right)$, and through (4) the instantaneous frequency component $f_{i, j}$ can be correlated with the amplitude $X\left(t_{i}, f_{i, j}\right)$ as follows:

$$
X\left(t_{i}, f_{i, j}\right)= \begin{cases}\left|x\left(t_{j}\right)\right|, & f_{i, j} \in\left[f_{d}, f_{u}\right] \\ 0 & \text { Others. }\end{cases}
$$


If the amplitude is correlated with the positive instantaneous frequency component, then, in the formula above, $f_{i, j}=$ $1 /\left(t_{j}-t_{i}\right), i \in[1, N]$ and $j \in[i, N]$, while if the amplitude is correlated with the reverse instantaneous frequency component, then, in the formula, $f_{i, j}=1 /\left(t_{i}-t_{j}\right), i \in[1, N]$ and $j \in(1, i]$.

Like the time-frequency spectrum of the time-frequency transformation, the processing result obtained from amplitude relevancy processing may also be expressed by the time-frequency spectrum; that is, the one-dimensional pulse sequence $\left\{x\left(t_{i}\right), i \in[1, N]\right\}$ is transformed into a twodimensional plane in which the amplitude $X\left(t_{i}, f_{i, j}\right)$ is expressed by the color space, with the pulse arrival time $t_{i}$ as horizontal coordinate and the instantaneous frequency $f_{i, j}$ as vertical coordinate. Figure 8 presents the time-frequency spectrum after the Steps (a)-(e) above are applied to Figure 7.

(f) Effective Instantaneous Frequency Extraction. After the amplitude relevancy processing above, the instantaneous frequency components $f_{i, j}$ extracted at the moment $t_{i}$ are not all effective instantaneous frequencies that help to diagnose the damage in the rolling bearing. Instead, they contain numerous ineffective instantaneous frequency components resulting from false interharmonic (see label $\mathrm{A}$ in Figure 8), cross-term interference (see label $\mathrm{B}$ in Figure 8), noise interference, wearout failure and poor lubrication inference (see label C in Figure 8), and other shock sources (see label D in Figure 8). Hence, it is necessary to weed out the ineffective instantaneous frequency components caused by these factors above, so as to highlight and extract the effective instantaneous frequency components.

(1) False Interharmonic Weeding-Out. The false interharmonic means the ineffective false instantaneous frequency component of the fraction times effective instantaneous frequency component produced by the reciprocal of the arrival time interval between several points on the pulse sequence with the time interval of $T_{1}$, after the pulse sequence containing the periodical sequence component with the time interval of $T_{1}$ experiences PATFTM.

The ineffective instantaneous frequency caused by the false interharmonic can be weeded out by following the false interharmonic criterion below.

False interharmonic criterion: taking the judgment as to whether the positive instantaneous frequency $f_{i, j}$ is a false interharmonic as an example, suppose that $\delta_{1}$ and $\delta_{2}$ are minor tolerances; if there is $k$ to make $\left|f_{i, j}-f_{i, k} / m\right|<\delta_{1}$ (wherein $f_{i, j} \neq 0,0<i<k<j<N$, and the variable $m$ is a positive integer and satisfies $1<m<j-k$, which is used to demonstrate that $f_{i, j}$ is an interharmonic of $f_{i, k}$ ) and $\left|w_{i, k}-w_{i, j}\right|<\delta_{2}\left(w_{i, j}\right.$ and $w_{i, k}$ denote the pulse width at the moments $t_{j}$ and $t_{k}$, resp.), then $f_{i, j}$ is an ineffective instantaneous frequency that is usually produced by the false interharmonic.

(2) Removal of Cross-Term Interference and Noise Interference. The cross-term interference means the ineffective instantaneous frequency component derived from the reciprocal of the arrival time interval between the staggered pulses

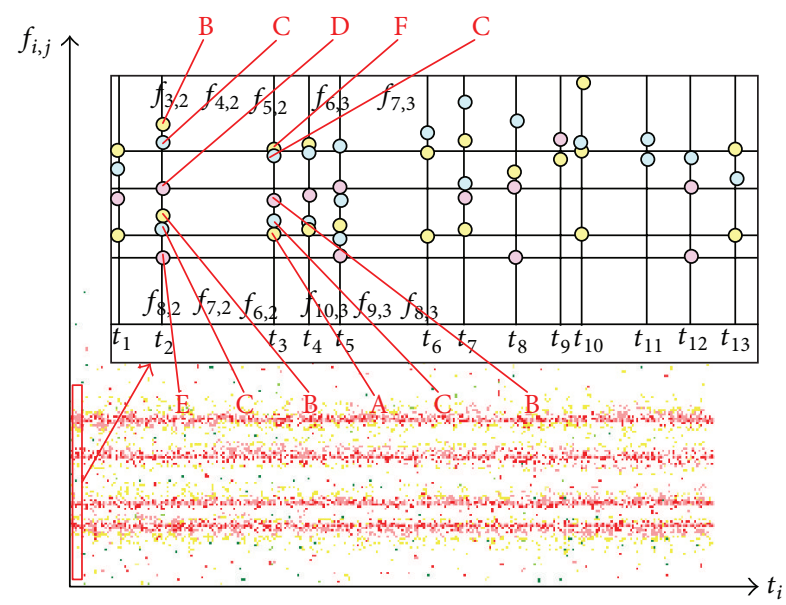
A: false interharmonic
D: other shock sources frequency
B: cross-term interference
E: fault feature frequency
C: noise interference, wearout failure, F: false interharmonic of other and poor lubrication inference shock sources frequency

FIGURE 8: Schematic diagram of amplitude relevancy processing.

comprising several effective instantaneous frequency components, after the pulse sequence containing several effective instantaneous frequency components, experiences PATFTM.

The noise interference means the ineffective instantaneous frequency component comes from the reciprocal of the arrival time interval between the different noise pulses as well as between the noise pulse and other pulses, after the pulse sequence containing noise pulse experiences PATFTM.

As the rolling bearing fault feature frequencies can be calculated ahead, by defining the frequency domain bandwidth $\left[f_{d}, f_{u}\right]$ of the instantaneous frequency component $f_{i, j}$, the ineffective instantaneous frequencies produced by the cross-term interface and noise interface, which fall out of the frequency domain bandwidth, can be preliminarily removed.

The ineffective instantaneous frequencies produced by the cross-term interface and noise interface, which fall within the frequency domain bandwidth, can be weeded out by following the cross-term and noise interference criterion.

Cross-term and noise interference criterion: suppose that $\delta_{3}$ is a minor tolerance, if there is no $k$ to make $\left|f_{i, j}-f_{i, k}\right|<$ $\delta_{3}$ (wherein $f_{i, j} \neq 0,0<k<i<j<N$ ), then $f_{i, j}$ is an ineffective instantaneous frequency that is usually produced by the cross-term and noise interference.

This is because the positive instantaneous frequency component and the reverse instantaneous frequency component are obtained from the same pulse sequence in the same way, while the only difference between them lies in the direction of the calculation adopted in obtaining the instantaneous frequency components. As the shock pulse produced by the cross-term and noise interference within the same pulse sequence takes place at random (excluding the cross-term interference caused by several effective instantaneous frequency components in which the common multiple relationship exists), the instantaneous frequency components $f_{i, j}$ and $f_{i, k}$ produced by the cross-term and noise interference within the positive instantaneous frequency components 


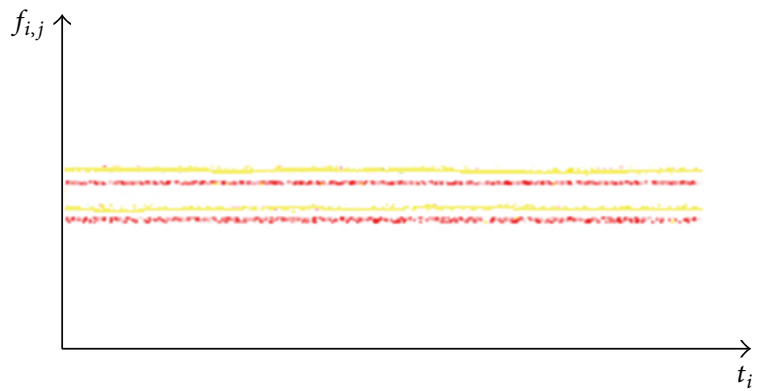

FIGURE 9: Schematic diagram of extraction of effective instantaneous frequency components.

and the reverse instantaneous frequency components are necessarily irrelevant, and as a rule, no $k$ is available for making $\left|f_{i, j}-f_{i, k}\right|<\delta_{3}$ (wherein $f_{i, j} \neq 0,0<k<$ $i<j<N$ ). However, for the shock pulse produced by the rolling bearing damage within the same pulse sequence, its occurring rule remains unchanged within a short time, and a certain phase shift alone takes place. Therefore, the changing rules of the instantaneous frequency components $f_{i, j}$ and $f_{i, k}$ produced by the rolling bearing damage within the positive instantaneous frequency components and the reverse instantaneous frequency components remain the same within a short time, and there is generally $k$ available for making $\left|f_{i, j}-f_{i, k}\right|<\delta_{3}$ (wherein $f_{i, j} \neq 0,0<k<i<j<N$ ). Hence, the cross-term and noise interference criterion may be followed for weeding out the ineffective instantaneous frequencies, without excluding those instantaneous frequency components arising from the rolling bearing damage.

(3) Removal of Wearout Failure and Poor Lubrication Interference. The wearout failure and poor lubrication interference mean the ineffective instantaneous frequency component produced by the arrival time interval between the different shock pulses resulting from wearout failure and poor lubrication as well as between the shock pulse and other pulses, after the pulse sequence containing the shock pulse arising from wearout failure and poor lubrication, experiences PATFTM.

The removal process of the wearout failure and poor lubrication interference is similar to that of the noise interference, and so the description thereof is here omitted.

(4) Removal of Other Shock Sources. The ineffective instantaneous frequency components produced by other shock sources, if not falling within the set frequency domain bandwidth $\left[f_{d}, f_{u}\right]$, may be directly removed. Or else, like the rolling bearing damage, they will produce the fault feature frequencies of specific physical significance and may be weeded out by analyzing the fault feature frequencies.

In general, when the effective instantaneous frequency components are extracted, the smaller $\delta_{1}, \delta_{2}$, and $\delta_{3}$ value adopted leads to the greater time resolution and frequency resolution of the time-frequency spectrum obtained. The results of extraction of the effective instantaneous frequency components from the time-frequency spectrum as shown in Figure 8 are shown in Figure 9.

To sum up, the process of the amplitude threshold processing, shock pulse ordering, instantaneous pulse ordering, plane transformation of instantaneous frequency, amplitude relevancy processing, and effective instantaneous frequency extraction is referred to as PATFTM.

After experiencing PATFTM, the instantaneous frequency component $f_{i, j}$ and its amplitude $X\left(t_{i}, f_{i, j}\right)$ directly reflect the time change feature of the analyzed vibration signals, and they can be used for real-time and quantitative fault diagnosis for the rolling bearing.

In addition, as the instantaneous frequency component $f_{i, j}$ is indirectly calculated from the pulse arrival time, the maximum resolution of time and frequency of the time-frequency spectrum obtained from PATFTM is no longer bound by Heisenberg's uncertainty principle [22] but depends upon the measuring precision of the pulse arrival time $t_{i}$.

\section{Simulation and Application Analysis}

4.1. Analysis of Simulation Signals. If any local damage comes into existence in the inner ring, outer ring, or rolling elements of the rolling bearing, the signal demodulated by SPM (i.e., the envelope signal obtained after band-pass filtering) may contain the periodical shock components. Besides, if these signals so demodulated also contain the strong background noise or other shock sources, then SPM will cause an erroneous diagnosis. However, PATFTM can extract the fault feature well and complete the fault diagnosis. Therefore, in the following the simulation signal $x(t)$, as shown in (5), containing the signal components produced by the inner ring damage, strong background noise, and other shock sources is made, and then it is processed by SPM, shock pulse frequency spectrum analysis method, shock pulse time-frequency analysis method, and PATFTM, respectively. The results of analysis are then compared, to validate the effectiveness of PATFTM:

$$
x(t)=x_{1}(t)+x_{2}(t)+x_{3}(t),
$$

where $x_{1}(t)$ is the simulated shock signal caused by the inner ring damage; $x_{2}(t)$ is the simulated shock signals caused by other shock sources; $x_{3}(t)$ is the simulated Gaussian white noise, with the mean value being $0 \mathrm{~mm} \cdot \mathrm{s}^{-2}$ and the variance being $1.1 \times 10^{-4} \mathrm{~mm} \cdot \mathrm{s}^{-2}$.

$x_{1}(t)$ may be expressed as the following pulse shock signals of periodical exponential attenuation, with the period being $T_{1}$ :

$$
\begin{gathered}
x_{1}(t)=\sum_{k=-\infty}^{k=+\infty} A_{1} e^{-\alpha_{1}\left(t-k T_{1}\right)} \cos \left[2 \pi f_{1}\left(t-k T_{1}\right)+\varphi_{1 k}\right] \\
\cdot U\left(t-k T_{1}\right),
\end{gathered}
$$

where $A_{1}$ denotes the peak amplitude and equals $0.05 \mathrm{~mm} \cdot \mathrm{s}^{-2} ; \alpha_{1}$ denotes the damping factor and equals $0.01 ; f_{1}$ denotes the resonance frequency and equals $30,000 \mathrm{~Hz} ; \varphi_{1 k}$ denotes the initial phase, falling within $\left[0^{\circ}\right.$, $360^{\circ}$ ]; $U(t)$ denotes the unit step function; $T_{1}$ denotes the period of occurrence of shock and equals $0.0075 \mathrm{~s}$. 
$x_{2}(t)$ may be expressed as the following pulse shock signals of periodical exponential attenuation, with the period being $T_{2}$ :

$$
\begin{aligned}
& x_{2}(t)=\sum_{k=-\infty}^{k=+\infty} A_{2} e^{-\alpha_{2}\left(t-k T_{2}\right)} \cos \left[2 \pi f_{2}\left(t-k T_{2}\right)+\varphi_{2 k}\right] \\
& \cdot U\left(t-k T_{2}\right),
\end{aligned}
$$

where $A_{2}$ denotes the peak amplitude and equals $0.07 \mathrm{~mm} \cdot \mathrm{s}^{-2} ; \alpha_{2}$ denotes the damping factor and equals $0.02 ; f_{2}$ denotes the resonance frequency and equals $30,000 \mathrm{~Hz} ; \varphi_{2 k}$ denotes the initial phase, falling within $\left[0^{\circ}\right.$, $\left.360^{\circ}\right] ; U(t)$ denotes the unit step function; $T_{2}$ denotes the period of occurrence of shock and equals $0.005 \mathrm{~s}$.

Figure 10(a) shows the time domain waveform of $x(t)$. It indicates that both positive transition and negative transition take place in the sporadic high frequency fading signal, and the initial phase changes with time. No periodical component representing the fault feature can be seen in the time domain waveform, and thus the fault diagnosis is impossible. Figure 10(b) shows the amplitude spectrum of the simulation signal $x(t)$.

Figure $10(\mathrm{c})$ shows the results of processing of the simulation signal $x(t)$ by SPM. As there is strong background noise, the statistical values $\mathrm{dBm}$ and $\mathrm{dBc}$ are generally high, as shown in the figure. If, based on this, it is judged that the rolling bearing is not well lubricated, it is obviously an erroneous diagnosis.

Figure 10(d) shows the frequency spectrum obtained after the simulation signal $x(t)$ is processed by shock pulse frequency spectrum analysis method. As shown in the figure, the visible feature frequencies are seen around $131.80 \mathrm{~Hz}$ and at its multiple frequency (corresponding to the shock signal with the period of $0.0075 \mathrm{~s}$ ) and around $200.20 \mathrm{~Hz}$ and at its multiple frequency (corresponding to the shock signal with the period of $0.005 \mathrm{~s}$ ). Based on this, it can be correctly judged that a fault happens. However, as the discrete sampling and interception of the continuous signal cause the frequency spectrum leakage and barrier effect, the feature frequencies extracted by shock pulse frequency spectrum analysis method have some errors. Besides, the method cannot display the shock occurring time. In addition, the method also produces the unwanted $66.89 \mathrm{~Hz}$ difference frequency signal component.

Figure 10(e) shows the time-frequency spectrum obtained after the simulation signal $x(t)$ is processed by shock pulse time-frequency analysis method, with the short-time Fourier transform being used and the $0.16 \mathrm{~s}$ wide Gaussian window being selected. As shown in the figure, within the time period analyzed, the visible feature frequencies are seen around $131.80 \mathrm{~Hz}$ and at its multiple frequency (corresponding to the shock signal with the period of $0.0075 \mathrm{~s}$ ) and around $200.30 \mathrm{~Hz}$ and at its multiple frequency (corresponding to the shock signal with the period of $0.005 \mathrm{~s}$ ). Based on this, it can be correctly judged that a fault happens. In comparison with shock pulse frequency spectrum analysis method, time-frequency analysis method can analyze two dimensions, that is, time and frequency, at the same time. However, as the method is restricted by the Heisenberg uncertainty principle, a certain width is present in the feature lines as shown in Figure 10(e), and the high time resolution and frequency resolution cannot be simultaneously achieved. Besides, the instantaneous feature frequencies extracted in this way have some resolution errors, and the shock sequence causes too many feature lines, which are thus not eye-striking enough.

Figure 10(f) shows the time-frequency spectrum obtained after the simulation signal $x(t)$ is processed by PATFTM (the amplitude threshold is set to be $0.03 \mathrm{~mm} \cdot \mathrm{s}^{-2}, f_{d} 0 \mathrm{~Hz}$, $f_{u} 500 \mathrm{~Hz}, \delta_{1} 1 \mathrm{~Hz}, \delta_{2} 0.00005 \mathrm{~s}$, and $\left.\delta_{3} 0.006 \mathrm{~mm} \cdot \mathrm{s}^{-2}\right)$. The visible feature frequencies are seen at $133.30 \mathrm{~Hz}$ and $200.00 \mathrm{~Hz}$ (corresponding to the shock signals with the periods of $0.0075 \mathrm{~s}$ and $0.005 \mathrm{~s}$, resp.), and thus the shock occurring frequency is accurately and clearly reflected. Based on this, it can be correctly judged that a fault happens. In contrast with the shock pulse frequency spectrum analysis method, which makes errors in the extracted feature frequencies due to such problems as frequency spectrum leakage and barrier effect and cannot supply high time resolution and frequency resolution at the same time owing to its restriction by the Heisenberg uncertainty principle, the PATFTM may, by setting minor tolerances $\delta_{1}, \delta_{2}$, and $\delta_{3}$, make the extracted instantaneous feature frequencies approach the theoretical value (as shown in Figure 10(f)) and provide high timefrequency resolution.

To sum up, PATFTM is capable of extracting the feature frequencies representing the rolling bearing damage effectively even in the presence of the strong background noise and other shock sources, and the feature frequency resolution supplied by the proposed method is higher than that by shock pulse frequency spectrum analysis method and shock pulse time-frequency analysis method.

To further validate the effectiveness of PATFTM and obtain the range of applicability of the method in the white noise environment, on the basis of the simulation signal $x_{1}(t)$, the simulation signals with different SNR $(0 \mathrm{~dB},-7 \mathrm{~dB}$, $-10 \mathrm{~dB}$, and $-11 \mathrm{~dB}$ ) are made and used to do a comparative analysis experiment with shock pulse time-frequency analysis method.

In the experiment, short-time Fourier transform with a 40.96 ms wide Gaussian window is used for shock pulse time-frequency analysis method, while the parameters of the amplitude threshold, $f_{d}, f_{u}, \delta_{1}, \delta_{2}$, and $\delta_{3}$, are set the same as the parameters adopted in Figure 10(f) for PATFTM. The experimental results are shown in Figure 11.

Figure 11(a) shows the time domain waveform of the simulation signals with different SNR as above, and Figures 11(b) and 11(c) show the results of processing of these simulation signals by shock pulse time-frequency analysis method and PATFTM. The results show that, with the decrease in SNR, PATFTM receives little effect. When SNR is $-10 \mathrm{~dB}$, the method remains able to suppress noise and extract the instantaneous feature frequencies with high time resolution and frequency resolution. However, when SNR is $-7 \mathrm{~dB}$, the white noise will interfere with the performance of shock pulse time-frequency analysis method. Although the frequency resolution of the method can be improved through increasing 


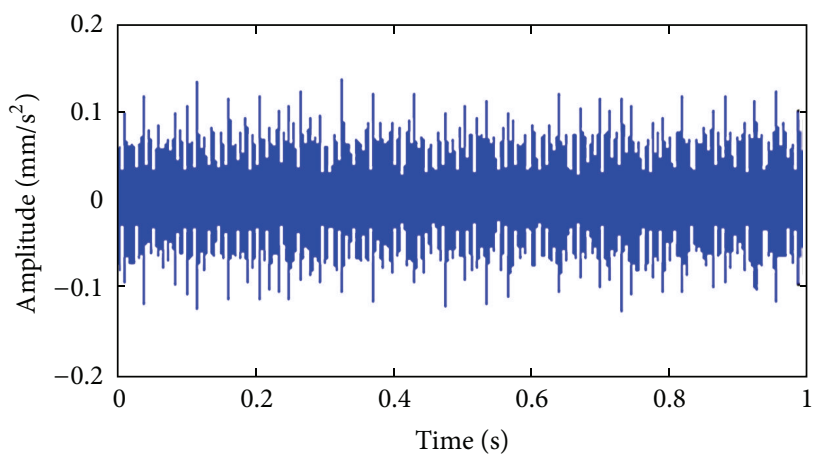

(a)

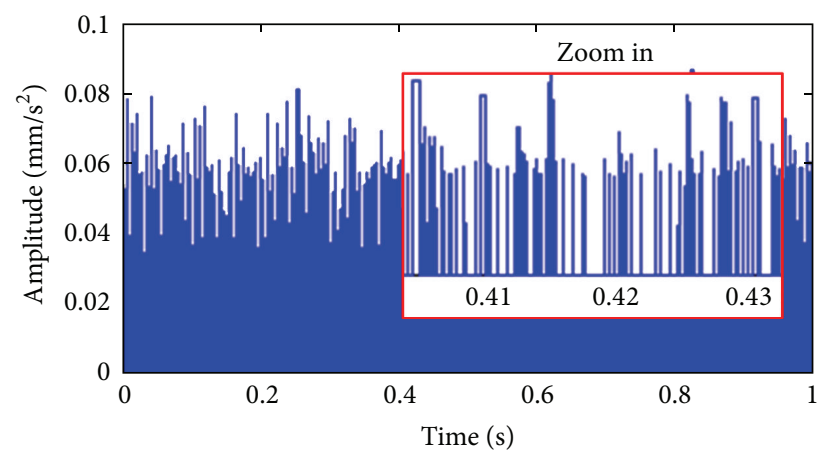

(c)

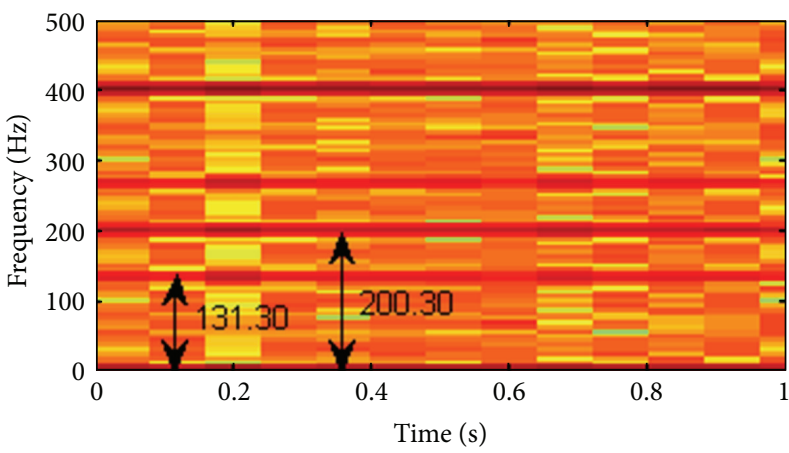

(e)

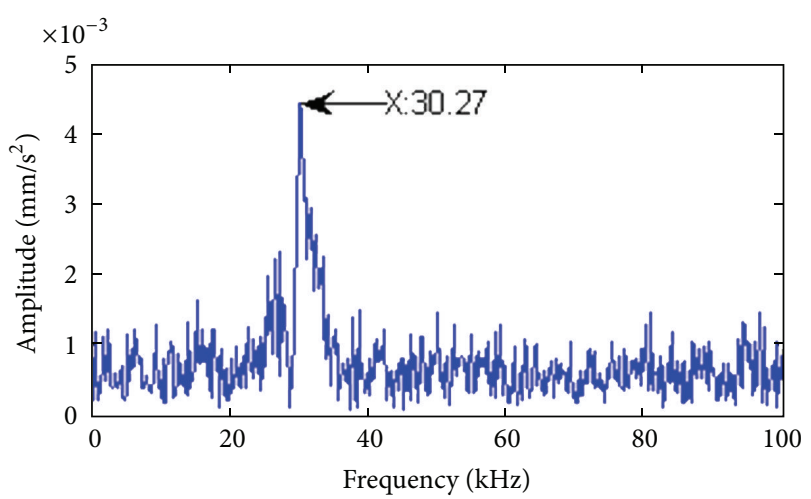

(b)

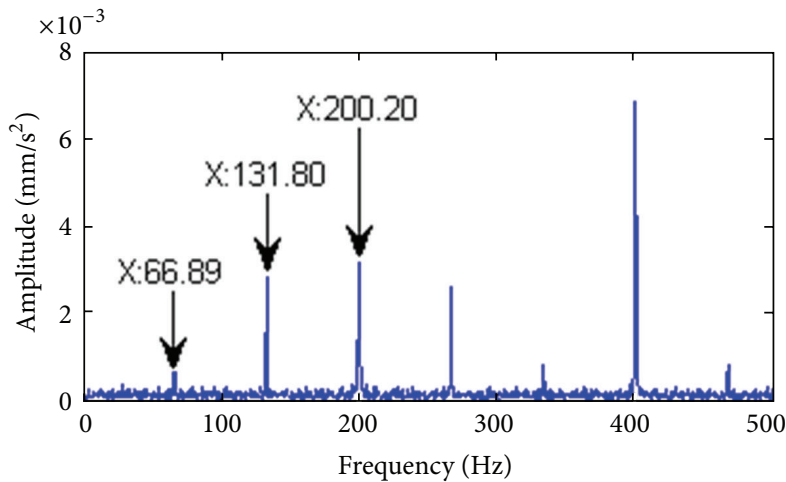

(d)

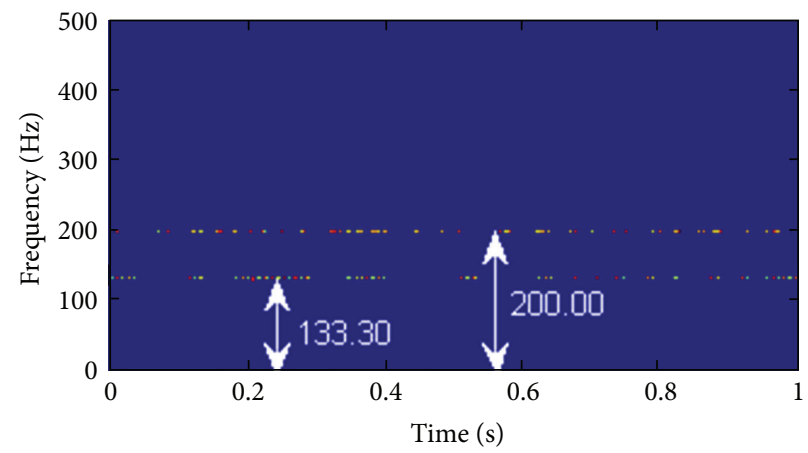

(f)

Figure 10: (a) The time domain waveform of $x(t)$; (b) the amplitude spectrum of $x(t)$; (c) the processing result of $x(t)$ by SPM; (d) the processing result of $x(t)$ by shock pulse frequency spectrum analysis method; (e) the processing result of $x(t)$ by shock pulse time-frequency analysis method; (f) the processing result of $x(t)$ by PATFTM.

the window width, the time resolution of the method will be lost.

In conclusion, the antinoise performance of the proposed method is much better than that of shock pulse timefrequency analysis method. Compared with shock pulse time-frequency analysis method, PATFTM can effectively suppress noise and extract the instantaneous feature frequencies with high time resolution and frequency resolution.

4.2. Analysis of Measured Data. To further validate the feasibility of PATFTM, the method is employed for processing and analyzing the measured data, and the results of processing and analysis are then compared with those of SPM, shock pulse frequency spectrum analysis method, and shock pulse time-frequency analysis method. Figure 12(a) shows the time domain waveform of the acceleration signal acquired by the shock pulse sensor at the pedestal of the Timken 566 bearing with a faulty inner ring. Figure 12(b) shows the time domain waveform of the envelope signal obtained after measured data was demodulated. In the test, the device's rotation frequency is $2.0417 \mathrm{~Hz}$. With reference to the structural parameters of the bearing and through the theoretical calculation [11], the fault feature frequency of the cage, rolling elements, outer ring, and inner ring of the bearing is obtained, that is, $0.89 \mathrm{~Hz}, 7.58 \mathrm{~Hz}, 17.77 \mathrm{~Hz}$, and $23.06 \mathrm{~Hz}$, respectively. Figures $12(\mathrm{c}), 12(\mathrm{~d}), 12(\mathrm{e})$, and $12(\mathrm{f})$ show the results of processing 

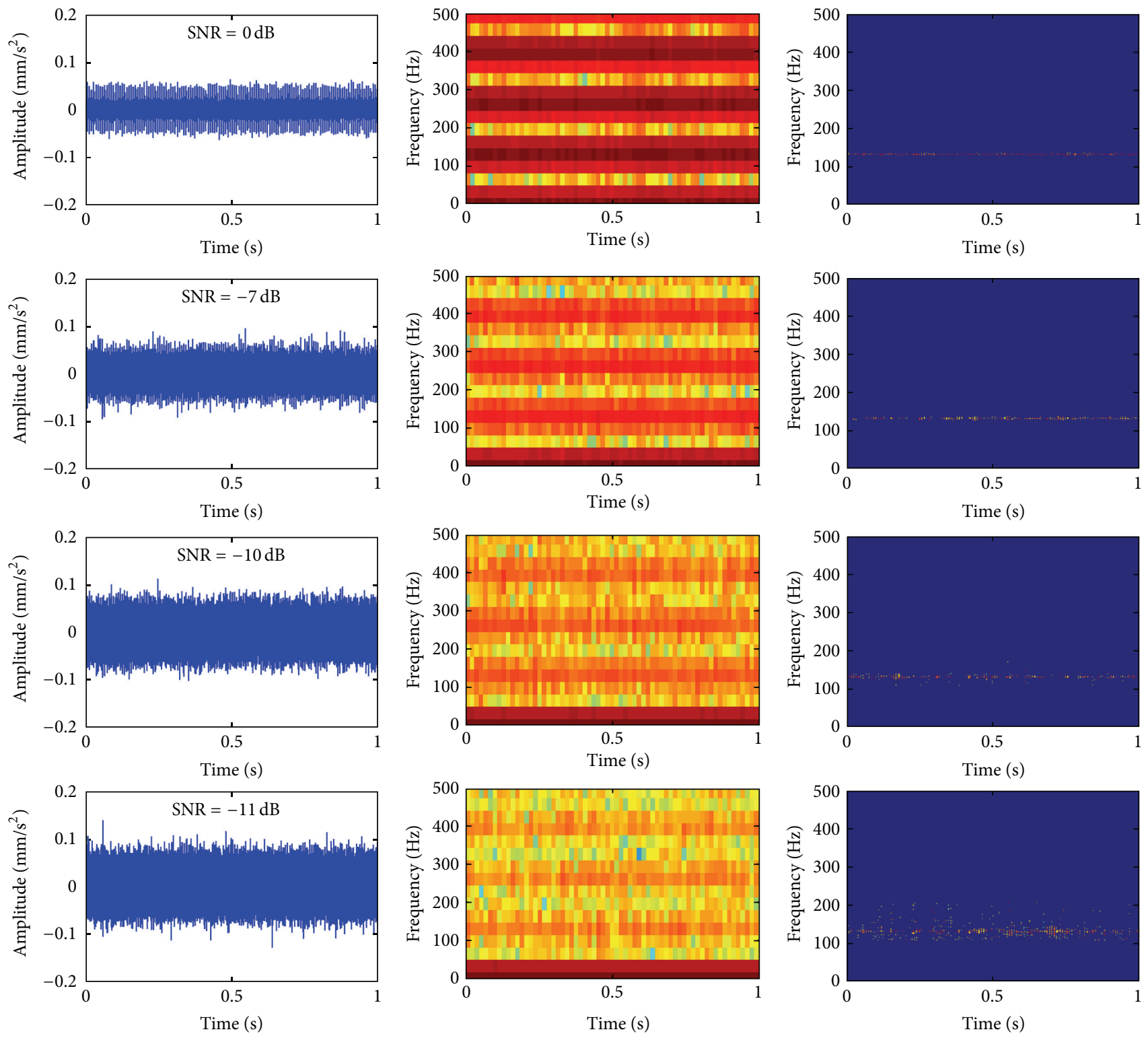

(a)

(b)

(c)

FIGURE 11: (a) The time domain waveforms of $x_{1}(t)$ with different SNR; (b) the processing results of $x_{1}(t)$ with different SNR by shock pulse time-frequency analysis method; (c) the processing results of $x_{1}(t)$ with different SNR by PATFTM.

of the signal as shown in Figure 12(a) by SPM, shock pulse frequency spectrum analysis method, shock pulse timefrequency analysis method, and PATFTM.

As shown in Figure 12(c), the periodical peak value takes place, and the value is great. So it can be judged that the bearing is damaged; however, which part of the bearing is damaged cannot be further judged. A series of $22.25 \mathrm{~Hz}$ harmonic frequencies and $22.25 \mathrm{~Hz}$ sideband frequencies, as shown in Figure 12(d), are close to the fault feature frequency of the inner ring obtained through theoretical calculation. Based on this, it can be judged that the inner ring of the bearing is damaged. In the frequency spectrum as shown in both Figures 12(e) and 12(f), the visible feature frequencies arise around $22.25 \mathrm{~Hz}$, which are close to the fault feature frequency of the inner ring obtained through theoretical calculation. So it can be judged that the inner ring of the bearing is damaged. However, with the almost same time resolution, Figure 12(f) can highlight the feature frequencies better than Figure 12(e) does, as well as with more accurate fault feature frequency extracted and higher resolution.

To sum up, PATFTM can be used for processing and analyzing the measured data well and extracting the fault feature frequencies representing the rolling bearing damage with high time resolution and frequency resolution.

\section{Conclusions}

(1) Conclusion of theoretical study analysis: PATFTM performs such processes as amplitude threshold processing, shock pulse ordering, instantaneous frequency calculation, 


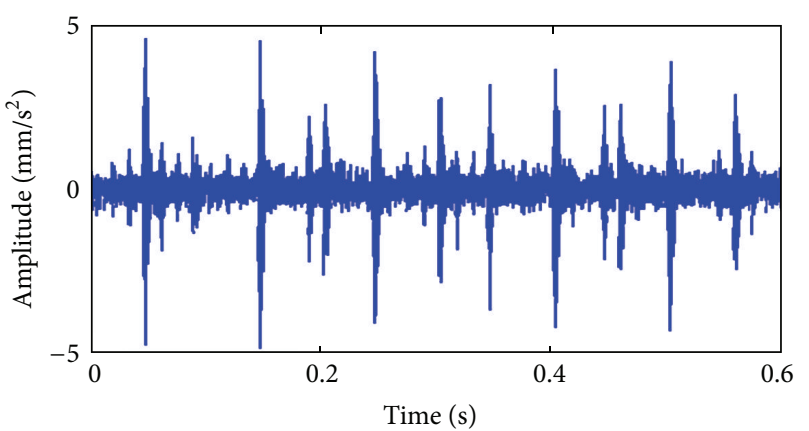

(a)

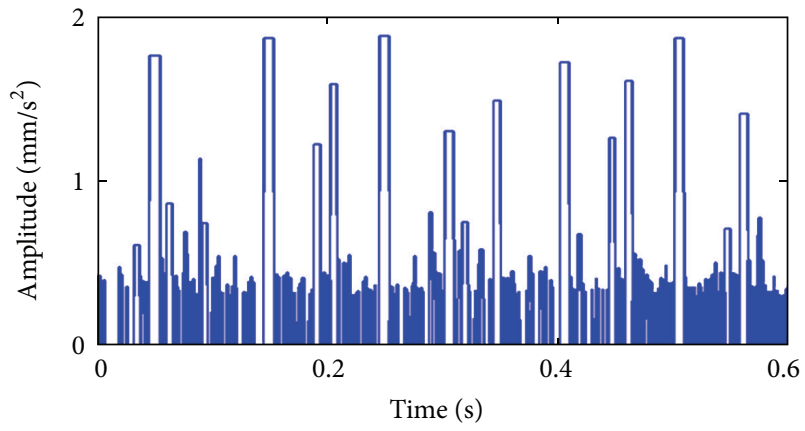

(c)

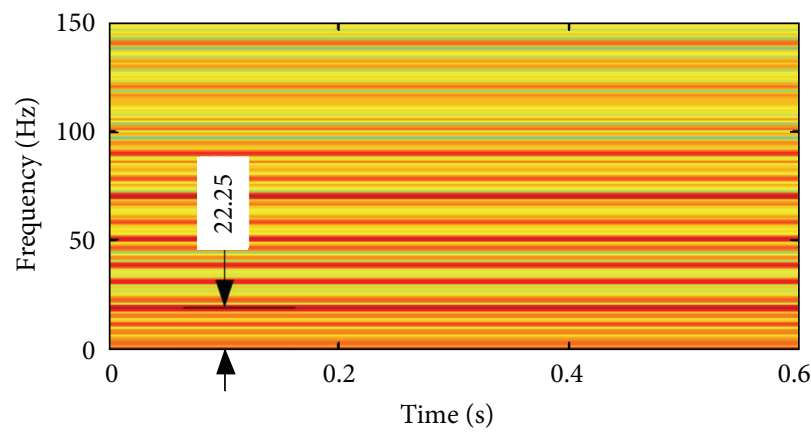

(e)

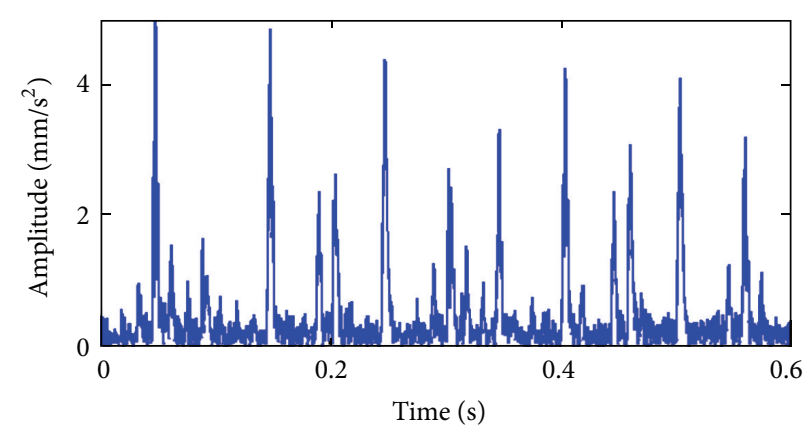

(b)

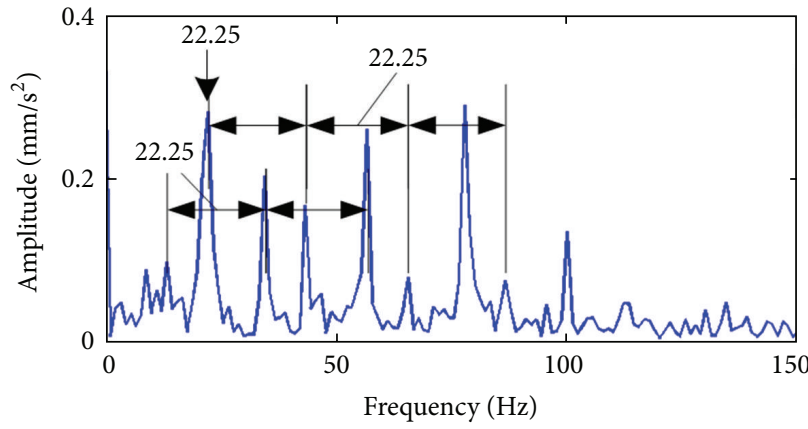

(d)

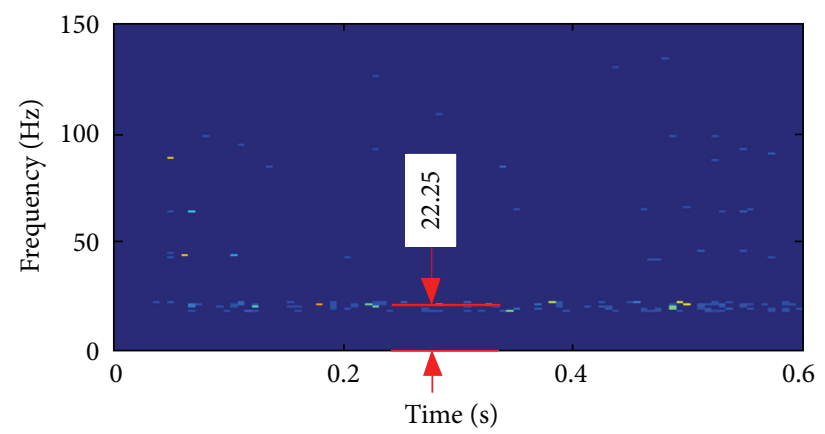

(f)

Figure 12: (a) The time domain waveform of the measured data; (b) the time domain waveform of the envelope signal of the measured data; (c) the processing result of the measured data by SPM; (d) The processing result of the measured data by shock pulse frequency spectrum analysis method; (e) the processing result of the measured data by shock pulse time-frequency analysis method; (f) the processing result of the measured data by PATFTM.

plane transformation of instantaneous frequency, and amplitude relevancy processing to the shock pulses extracted by SPM and transforms the shock pulse sequence of one-dimensional time domain into the two-dimensional time-frequency domain. Through the extraction of the effective instantaneous frequencies, it excludes the influences from other interference factors and highlights and extracts the fault feature representing the rolling bearing damage. The results of theoretical analysis show that the maximum time and frequency resolution of PATFTM is no longer restricted by the Heisenberg uncertainty principle but depends upon the measuring precision of the pulse arrival time. PATFTM can obtain the time-frequency spectrum with the high time and frequency resolution, and by extracting the effective instantaneous frequencies, it can greatly suppress the noises, accurately extract the feature frequencies, and complete the rolling bearing fault diagnosis as desired.

(2) Conclusion of simulation experiment analysis: the results of the comparative simulation experiment in which the simulation signal produced by the inner ring damage, strong background noise, and other shock sources is made show that PATFTM is able to effectively extract the feature frequencies representing the rolling bearing damage even in the presence of the strong background noise and other shock sources, and the feature frequency resolution supplied by the method is higher than that by shock pulse frequency spectrum analysis method and shock pulse time-frequency analysis method. The results of the simulation experiment in which the simulation signals with different SNR are made show that PATFTM is little affected by noises and that when the SNR is $-10 \mathrm{~dB}$, the method can still suppress noises well 
and extract the instantaneous feature frequencies with high time resolution and frequency resolution.

(3) Conclusion of measured data analysis: the results of measured data analysis show that PATFTM can effectively extract the fault feature representing the rolling bearing damage, and the extracted fault feature frequency accords with the theoretically calculated fault feature frequency. In addition, the same conclusion as that drawn in the simulation experiment is reached; that is, the resolution of the feature frequencies extracted by PATFTM is superior to that by shock pulse frequency spectrum analysis method and shock pulse time-frequency analysis method.

To sum up, the results of simulation and application analysis come in agreement with the results of theoretical analysis, and they both show that PATFTM can effectively suppress noises, highlight the feature frequencies, and avoid erroneous diagnosis. Thus, it is an effective method for extracting the rolling bearing fault features with high time and frequency resolution.

However, in the presence of strong background noise, although PATFTM is able to accurately diagnose the rolling bearing damage, it cannot qualitatively diagnose the wearout failure and poor lubrication in the rolling bearing. For this reason, PATFTM needs to be further studied and perfected.

\section{Competing Interests}

The authors declare no competing interests.

\section{Acknowledgments}

This work was supported in study design by the National Natural Science Foundation of China (Grant no. 51275546) and the Natural Science Foundation Project of Chongqing CSTC (Grant no. CQ cstc2011jjjq70001).

\section{References}

[1] F. Zhang and K. Ding, "Research on the three algorithms and limitations of generalized detection-filtering demodulation analysis," Journal of Vibration Engineering, vol. 15, no. 2, pp. $243-$ 248, 2002.

[2] Y. B. Li, M. Q. Xu, H. Y. Zhao, Y. Wei, and W. Huang, "A new rotating machinery fault diagnosis method based on improved local mean decomposition," Digital Signal Processing, vol. 46, pp. 201-214, 2015.

[3] C. T. Yiakopoulos and I. A. Antoniadis, "Wavelet based demodulation of vibration signals generated by defects in rolling element bearings," Shock and Vibration, vol. 9, no. 6, pp. 293306, 2002.

[4] R. Yan, R. X. Gao, and X. Chen, "Wavelets for fault diagnosis of rotary machines: a review with applications," Signal Processing, vol. 96, 15 pages, 2014.

[5] H. C. Wang, J. Chen, and G. M. Dong, "Feature extraction of rolling bearing's early weak fault based on EEMD and tunable Q-factor wavelet transform," Mechanical Systems and Signal Processing, vol. 48, no. 1-2, pp. 103-119, 2014.

[6] M. Zhao, J. Lin, X. Xu, and X. Li, "Multi-Fault detection of rolling element bearings under harsh working condition using imf-based adaptive envelope order analysis," Sensors, vol. 14, no. 11, pp. 20320-20346, 2014.

[7] Y.-T. Sheen, "On the study of applying Morlet wavelet to the Hilbert transform for the envelope detection of bearing vibrations," Mechanical Systems and Signal Processing, vol. 23, no. 5, pp. 1518-1527, 2009.

[8] Y. Qin, J. X. Wang, and B. P. Tang, "Multicomponent demodulation based on iterated Hilbert transform and its application," Journal of Mechanical Engineering, vol. 45, no. 8, pp. 37-44, 2009.

[9] J. R. Stack, R. G. Harley, and T. G. Habetler, "An amplitude modulation detector for fault diagnosis in rolling element bearings," IEEE Transactions on Industrial Electronics, vol. 51, no. 5, pp. 1097-1102, 2004.

[10] I. Moumene and N. Ouelaa, "Application of the wavelets multiresolution analysis and the high-frequency resonance technique for gears and bearings faults diagnosis," The International Journal of Advanced Manufacturing Technology, vol. 83, no. 5, pp. 1315-1339, 2016.

[11] S. Patidar and P. K. Soni, "An overview on vibration analysis techniques for the diagnosis of rolling element bearing faults," International Journal of Engineering Trends and Technology, vol. 4, no. 5, pp. 1803-1809, 2013.

[12] R. Yang, J. Kang, J. Zhao, J. Li, and H. Li, "A case study of bearing condition monitoring using SPM," in Proceedings of the Prognostics and System Health Management Conference (PHM '14), pp. 695-698, Zhangjiajie, China, August 2014.

[13] N. Tandon, G. S. Yadava, and K. M. Ramakrishna, "A comparison of some condition monitoring techniques for the detection of defect in induction motor ball bearings," Mechanical Systems and Signal Processing, vol. 21, no. 1, pp. 244-256, 2007.

[14] H. Q. Sang, "Application on bearing detection in coal mine production," Shanxi Coking Coal Science \& Technology, vol. 34, pp. 14-16, 2010.

[15] L. Zhen, H. Zhengjia, Z. Yanyang, and C. Xuefeng, "Bearing condition monitoring based on shock pulse method and improved redundant lifting scheme," Mathematics and Computers in Simulation, vol. 79, no. 3, pp. 318-338, 2008.

[16] D. E. Butler, "The Shock-pulse method for the detection of damaged rolling bearings," Non-Destructive Testing, vol. 6, no. 2, pp. 92-95, 1973.

[17] V. Barzdaitis, V. Žemaitis, K. Žebelys, Z. Pocius, and P. Mažeika, "Condition monitoring of roller bearings using different diagnostic methods," Diagnostyka, vol. 30, no. 1, pp. 53-60, 2004.

[18] Y. Zhang, L. Y. Lv, and S. T. Wan, "The shock pulse method applied on fault diagnosis of rolling bearing," Petro-Chemical Equipment Technology, vol. 28, no. 4, pp. 60-64, 2007.

[19] J. Yan, "Research on diagnosing the fault in rolling bearing based on shock pulse method," Compressor Blower \& Fan Technology, vol. 2, pp. 78-80, 2009.

[20] W.-H. Yang and M.-G. Gao, "Deinterleaving of pulse signal based on plane transformation," Transaction of Beijing Institute of Technology, vol. 25, no. 2, pp. 151-154, 2005.

[21] A. W. Ata'a and S. N. Abdullah, "Deinterleaving of radar signals and PRF identification algorithms," IET Radar, Sonar and Navigation, vol. 1, no. 5, pp. 340-347, 2007.

[22] R. Parhizkar, Y. Barbotin, and M. Vetterli, "Sequences with minimal time-frequency uncertainty," Applied and Computational Harmonic Analysis, vol. 38, no. 3, pp. 452-468, 2015. 


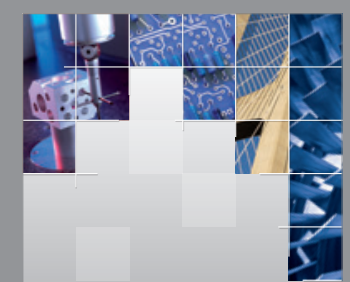

\section{Enfincering}
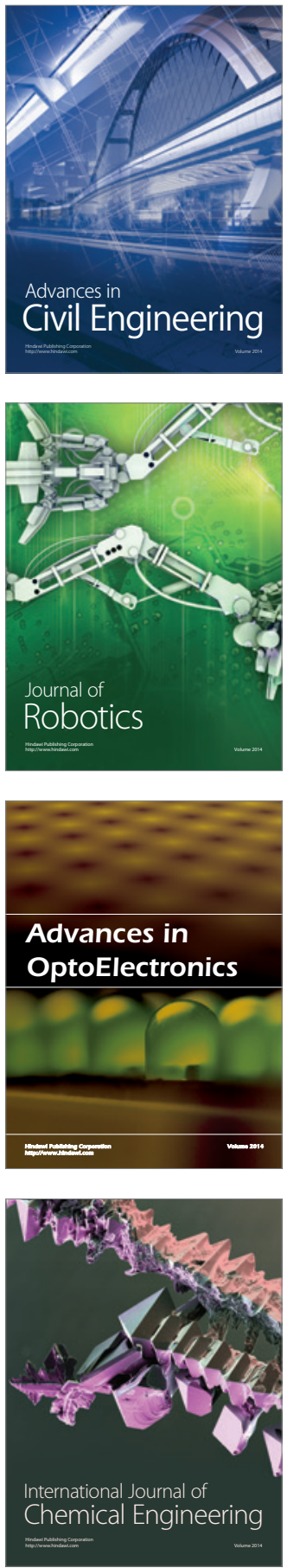

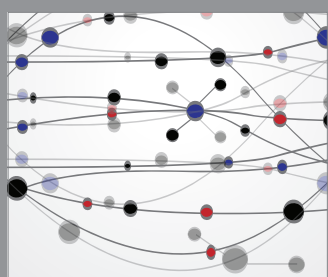

The Scientific World Journal

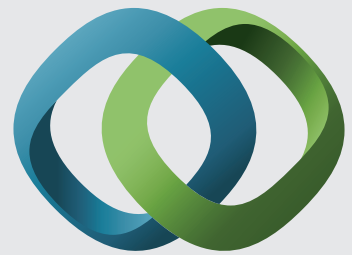

\section{Hindawi}

Submit your manuscripts at

http://www.hindawi.com
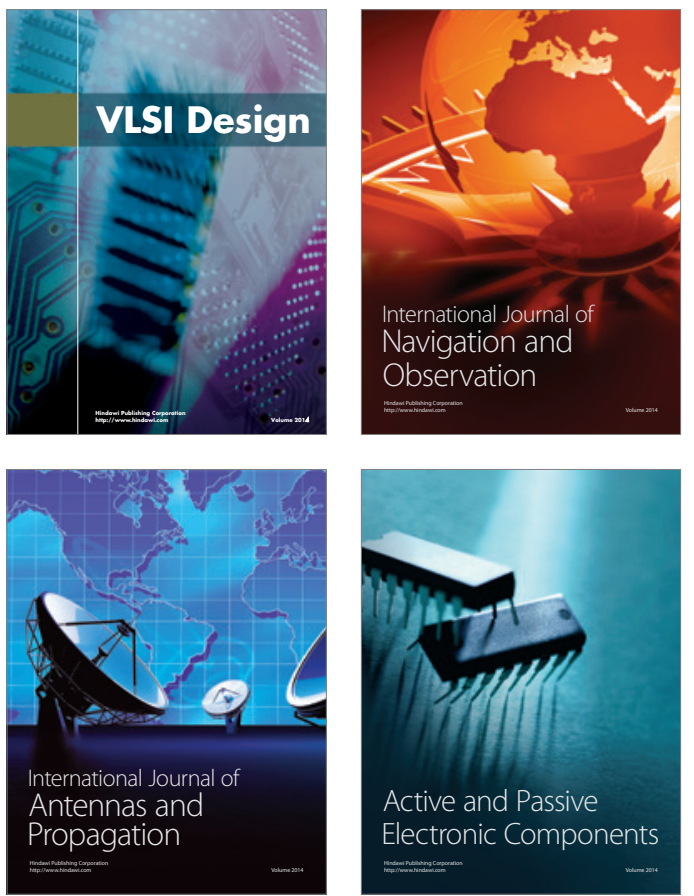
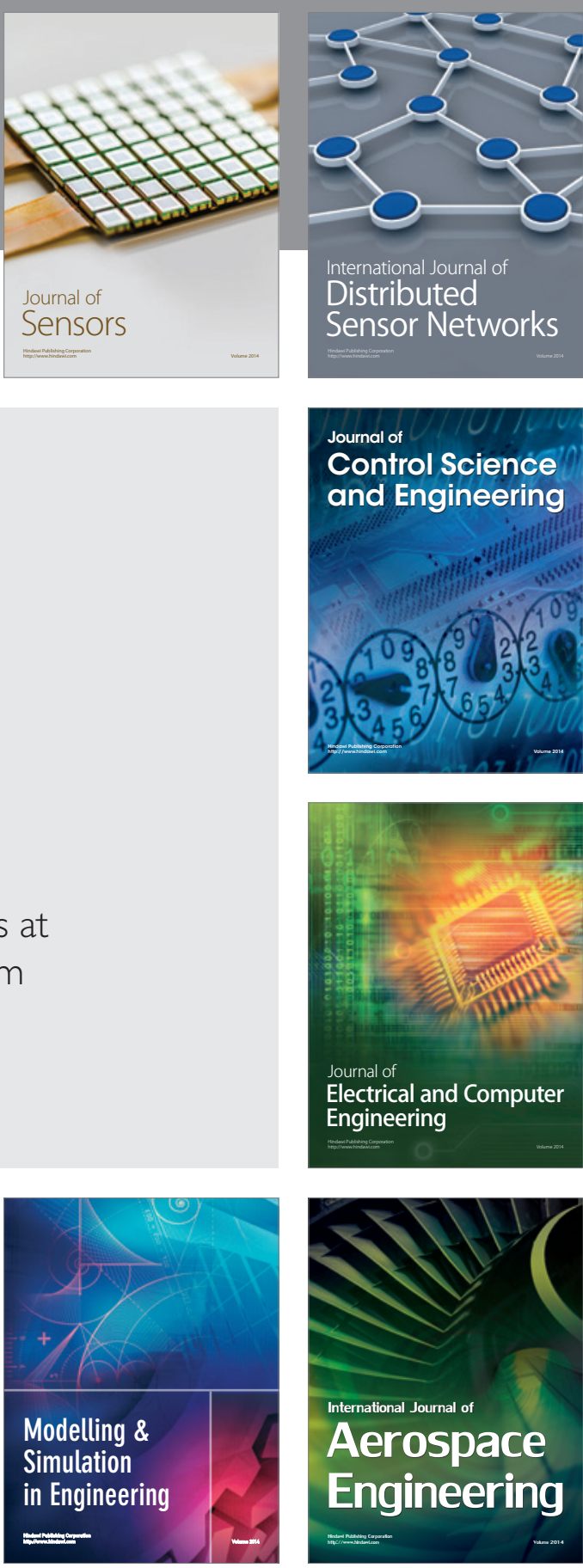

International Journal of

Distributed

Sensor Networks

Journal of

Control Science

and Engineering
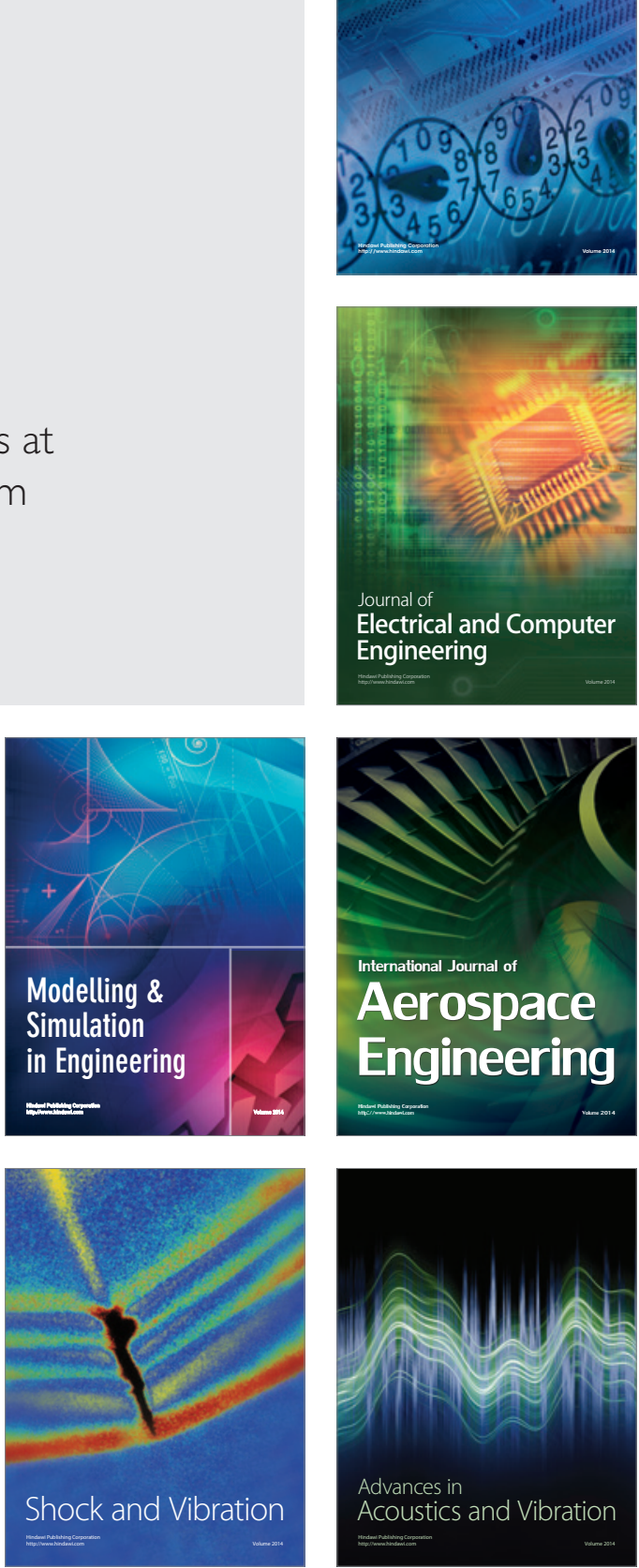